\title{
Lesiones por violencia familiar en el reconocimiento médico Legal (RML).
}

\author{
Ulises Mejía Rodríguez ${ }^{1}$, José Bolaños Cardozo², Alex Mejía Rodríguez ${ }^{3}$
}

\section{RESUMEN}

Objetivo: Determinar las lesiones por violencia familiar y sus características.

Material y Métodos: Se realizó un estudio descriptivo prospectivo de enero a diciembre del 2013.

Resultados: En el año 2013, se realizaron 1754 RML, de las cuales 423 correspondieron a lesiones por violencia familiar; el 30,97\% (131/423) correspondió a peritadas entre 36 a 49 años. Los hallazgos encontrados fueron: 93,14\%(394/423) fueron adultos. Respecto al agente causante. El 39,48\%(167/423) es producido por uña humana, en agredidos varones, y en mujeres, el 21,04\%(89/423) el agente contuso (puñete, patada, etc.) fue el agente causante, los casados y convivientes tienen mayor frecuencia de lesiones por violencia familiar, el 45,63\% (193/423) de las mujeres peritadas era casada, y en el sexo masculino el 23,40\% (99/423) eran casados; respecto a la región anatómica lesionada, el 8,75\%(37/423) de varones presentó lesiones en cabeza y cuello, entre otros hallazgos.

Conclusión: Las lesiones por violencia familiar son frecuentes en el sexo masculino como en el sexo femenino, las mujeres presentan lesiones por agente contuso debido a la fuerza que la caracterizaba a los agresores que vendrían a ser varones en la mayoría de casos. Los varones presentan lesiones por uña humana. (Horiz Med 2015; 15(1): 21-29)

Palabras clave: Lesiones, Lesiones por Violencia Familiar, Reconocimiento Médico Legal. (Fuente: DeCS BIREME).

Injury from domestic violence in the forensic examination (FE).

\begin{abstract}
Objective: To determine the injuries from domestic violence and their characteristics.

Material and Methods: A prospective descriptive study from January to December 2013.

Results: In 2013, 1754 FE were performed, of which 423 were for injuries from domestic violence; $30.97 \%(131 / 423)$ corresponded to patients between $36-49$ years. The findings were: $93.14 \%$ (394/423) were adults. Regarding the causative agent, 39.48\% (167/423) were produced by human nail in assaulted men and women, $21.04 \%(89 / 423)$ blunt agent (hitting, kicking, etc) was the causative agent, the married and cohabiting couples have increased frequency of domestic violence, 45.63\% (193/423) of women patients were married, and in males $23.40 \%(99 / 423)$ were married; concerning the injured anatomical region, $8.75 \%(37 / 423)$ of men had lesions in the head and neck and in females $8.98 \%(38 / 423)$.

Conclusion: domestic violence inj uries are common in males as in females, women have inj uries by blunt agents due to the force of male offenders in most cases. Men have human nail injuries. (Horiz Med 2015; 15(1): 21-29)
\end{abstract}

Key words: Injuries, Injuries by Domestic Violence, Forensic examination. (Source: MeSH NLM).

\footnotetext{
Médico Legista, Master en Medicina Forense, Doctorando en Criminalística.

Insituto de Medicina Legal Perú. Lima, Perú

Estudiante de Medicina Universidad Nacional Jorge Basadre - Tacna, Perú
} 


\section{INTRODUCCIÓN}

La violencia familiar no puede ser considerada como un fenómeno unitario, ya que es un problema emergente de salud pública; necesita un análisis multidisciplinario (médicos, abogados, psicólogos, etc.)(1), más aun cuando las víctimas con lesiones físicas y psicológicas denuncian el hecho ante las autoridades correspondientes.

La Declaración de las Naciones Unidas sobre Erradicación de la Violencia contra las Mujeres, adoptada por la Asamblea General de la ONU en 1993, proporciona un marco amplio y útil para definir la violencia contra la mujer pero para su monitoreo, se necesitarán definiciones operativas más específicas.

La violencia familiar abarca, entre otras, "la violencia física, sexual y psicológica que se produce en el seno de lafamilia, incluidaslaspalizas, el abuso sexual de niñas, la violencia relacionada con la dote, la violación marital, y otras prácticas tradicionales dañinas dentro del entorno familiar(2). El maltrato a menores en el entorno familiar entraría dentro de este último concepto, como aquellosactos que privan al niño o niña de sus derechos y libertades, e inciden negativamente en su bienestary desarrollo $(3,4)$.

La UNICEF (5), basándose en la Convención de Derechos del Niño, que en su Artículo 19, habla de la protección ante "cualquier forma de violencia física o mental", determina la necesidad de considerar maltrato, no solo la violencia directa, sino también los efectos indirectos de la violencia familiar sobre los niños; basándose en estudios acerca de la violencia intrafamiliar que han puesto de relieve losefectos psicológicos adversos que tienen sobre los niños presenciar o escuchar situaciones violentas (físicas o psicológicas) de sus padres.

En Hospitales en todo el Perú, los médicos atienden a víctimas de violencia familiar, quienes Ilevan lesiones traumáticas de diferentes tipos, las cuales son consecuencia directa de la violencia dentro de la familia. Los médicos del Instituto de Medicina Legal, no escapan a ello, debido a que a diario se perennizan en los Reconocimientos Médico Legales (RML) las lesiones producidas por este problema emergente de salud pública que no solo se suscita en el Perú, sino que es un problema de Salud a nivel mundial, el cual no distingue sexo, estado civil, nivel socio económico ni socio cultural.

Las autoridades que solicitan un Reconocimiento Médico Legal (PNP, Fiscalías de Familia, Fiscalías Penales, Poder Judicial, DEMUNA, Centro de Emergencia Mujer, INABIF, entre otros) solicitan a los médicos legistas una descripción detallada de las lesiones, donde se debe concluir los agentes causantes (objetos contusos, agentes cortantes, uña humana, arma blanca, proyectil de arma de fuego, etc).

Además concomitantemente la autoridad solicitante debe acompañar a la solicitud de RML por Violencia Familiar otra solicitud para Pericia Psicológica, en la cual el Psicólogo Forense deberá establecer si existe o no daños psicológicos, y el impacto social que ha producido este comportamiento en la agraviada víctima de violencia familiar $(6,7)$.

Los médicos, más aun si son médicos legistas, deberán diferenciar las características de las lesiones traumáticas que se presentan en víctimas de violencia familiar (8).

En el informe médico o Reconocimiento Médico Legal deberá incluir una valoración y cuantificación de las lesiones lo que se verá expresado en los días de incapacidad que requiere el peritado como en los días de atención facultativa que amerita la lesión encontrada en el RML, datos que serán de utilidad para la autoridad policial y/o fiscal, ya que ello será la prueba para poder formalizar la denuncia en la fiscalía de Familia dela provincia donde se ha suscitado el incidente.

El personal de la Salud, sobre todo el personal médico, de cada provincia debe saber la proyección legal y social de la evaluación de lesiones por violencia familiar y su perennización en el Reconocimiento Médico Legal, ya que la legislación peruana establece como obligación la certificación de lesiones traumáticas en ausencia de un médico legista (9), así como la notificación a la autoridad 
competente (10), lo que deberá cumplirse no sin antes capacitar al personal de la salud en identificación y descripción de las características de lesiones por violencia familiar (11). Posteriormente la autoridad solicitante generará la tipificación de faltas, lesiones leves o lesiones graves según sea el caso.

\section{MATERIAL Y MÉTODOS}

Estudio descriptivo prospectivo, realizado en la División Médico Legal Chucuito ubicada en la ciudad de Puno, durante los meses de Enero a Diciembre 2013. Para el estudio, se seleccionó las pericias médico legales de delitos contra la libertad sexual, las que fueron solicitadas por la PNP, Fiscalia y Juzgado.

Para proceder al examen médico legal de lesiones por violencia familiar, a la toma de fotografias, se solicitó autorización a través del formato de consentimiento informado a las personas examinadas; siendo asi y amparándonos en las consideraciones del acto médico y en el sigilio pericial omitimos la identificaciòn de las personas peritadas.

La población de estudio fueron todas las peritadas que denunciaban agresiones por violencia familiar y que acudieron a la DML I Chucuito, para un examen de reconocimiento médico legal por violencia familiar. Se determinó y describió las lesiones y caracteristicas de las lesiones; clasificando las lesiones por violencia familiar en: recientes $(<7$ días) y antiguas ( $>7$ días) dependiendo del tiempo de producidas.

Se excluyó del estudio a las personas que se negaron a la realización del examen de reconocimiento médico legal. Los datos fueron analizados con la ayuda del programa Microsoft Excel 2013.

\section{RESULTADOS}

Se realizaron 1754 Reconocimientos Médicos Legales (RML); de los cuales 2,67 \% (47/1754) fueron RML ginecológicos por delitos contra la libertad sexual, 24,12\% (423/1754) por violencia familiar, $36,20 \%(635 / 1754)$ por lesiones, 0,11\% (2/1754) por mordedura canina, $12,48 \%(219 / 1754)$ post facto, 2,05\% (36/1754) por suceso de tránsito, $17,45 \%(306 / 1754)$ pericias de psicología forense y el $4,92 \%$ otros.

El mayor porcentaje de las peritadas tenían entre 15 a 17 años, 38,29\% (18/47), seguido de peritadas entre 18 a 60 años de edad con 27,66\% (13/47). Figura 1.

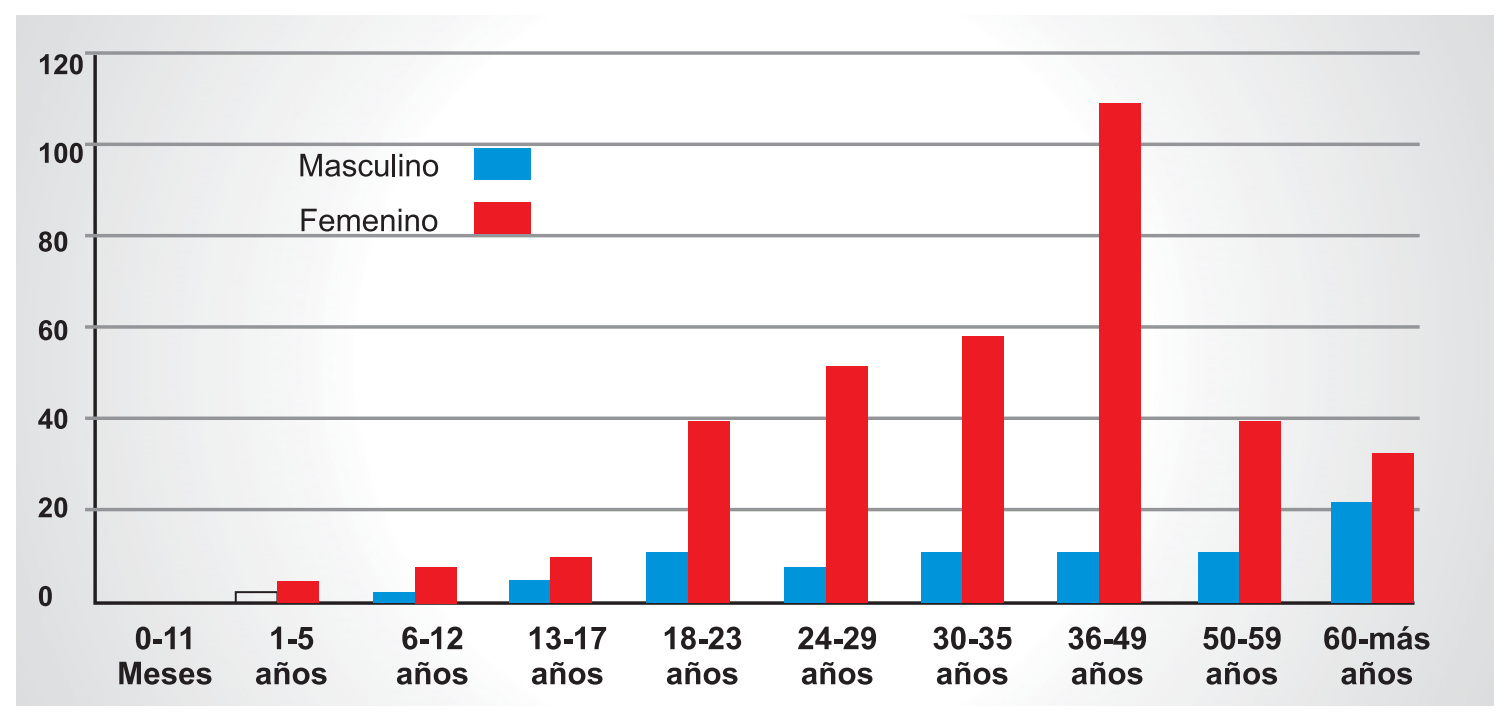

Figura 1.- Personas evaluadas por sexo y grupos etáreos en RML por Violencia Familiar. 
Tabla 1. Personas evaluadas por sexo y grupos etáreos en RML por Violencia Familiar.

\begin{tabular}{|c|c|c|c|c|c|}
\hline \multicolumn{6}{|c|}{ Lesiones por violencia familiar según sexo y grupos etareos } \\
\hline & Masculino & $\%$ & Femenino & $\%$ & Total \\
\hline 0-11 Meses & 0 & 0.00 & 0 & 0.00 & 0 \\
\hline 1-5 Años & 2 & 0.47 & 4 & 0.95 & 6 \\
\hline 6-12 Años & 2 & 0.47 & 7 & 1.65 & 9 \\
\hline 13-17 Años & 5 & 1.18 & 9 & 2.13 & 14 \\
\hline 18-23 Años & 10 & 2.36 & 36 & 8.51 & 46 \\
\hline 24-29 Años & 7 & 1.65 & 46 & 10.87 & 53 \\
\hline 30-35 Años & 10 & 2.36 & 53 & 12.53 & 63 \\
\hline 36-49 Años & 31 & 7.33 & 100 & 23.64 & 131 \\
\hline 50-59 Años & 14 & 3.31 & 37 & 8.75 & 51 \\
\hline 60- Mas Años & 20 & 4.73 & 30 & 7.09 & 50 \\
\hline Total & 101 & 23.88 & 322 & 76.12 & 423 \\
\hline
\end{tabular}

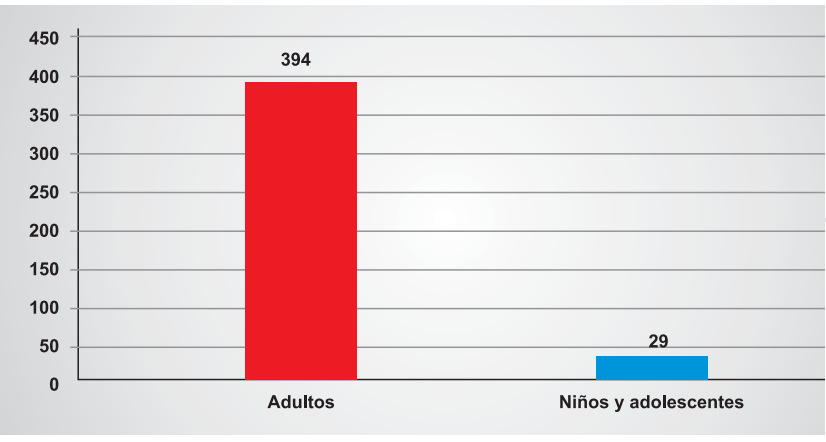

Figura 2.- Personas evaluadas según mayoria de edad, en RML por Violencia Familiar.
De las 423 evaluaciones por violencia familiar realizadas con el fin de determinar lesiones, el $93,14 \%(394 / 423)$ peritados eran adultos, siendo solo el 6,86\% ( 29/423) peritados niños y adolescentes. (Figura 2).

El agente causante de lesiones por violencia familiar varió segun el sexo. En varones, el 39,48\% (167/423) fue producido por uña humana, el 10,17\%(43/423) por un agente contuso. En las mujeres que fueron victima de violencia familiar, el $21,04 \%$ (89/423) tuvo como agente causante lesión por agente contuso, seguido con 7,57\% (32/423) por agente contuso cortante. (Tabla 2, Figura 3).

Tabla 2. Clasificacion de las lesiones según el agente que las produce y según el sexo de la victima de violencia familiar.

AGENTE QUE PRODUCE LA LESION

\begin{tabular}{lcccc} 
& \multicolumn{2}{c}{ Hombre } & \multicolumn{2}{c}{ Mujer } \\
\hline \multicolumn{1}{c}{ Agente } & Número & $\%$ & Número & $\%$ \\
\hline Contuso & 43 & 43 & 43 & 43 \\
Cortante & 7 & 7 & 7 & 7 \\
Contuso cortante & 15 & 15 & 15 & 15 \\
Arma de fuego & 2 & 2 & 2 & 2 \\
Agente constrictor & 9 & 9 & 9 & 9 \\
Uña humana & 167 & 167 & 167 & 167 \\
Mordedura humana & 5 & 5 & 5 & 5 \\
Otro agente & 4 & 4 & 4 & 4 \\
\hline Total & 252 & 252 & 252 & 252 \\
\hline
\end{tabular}




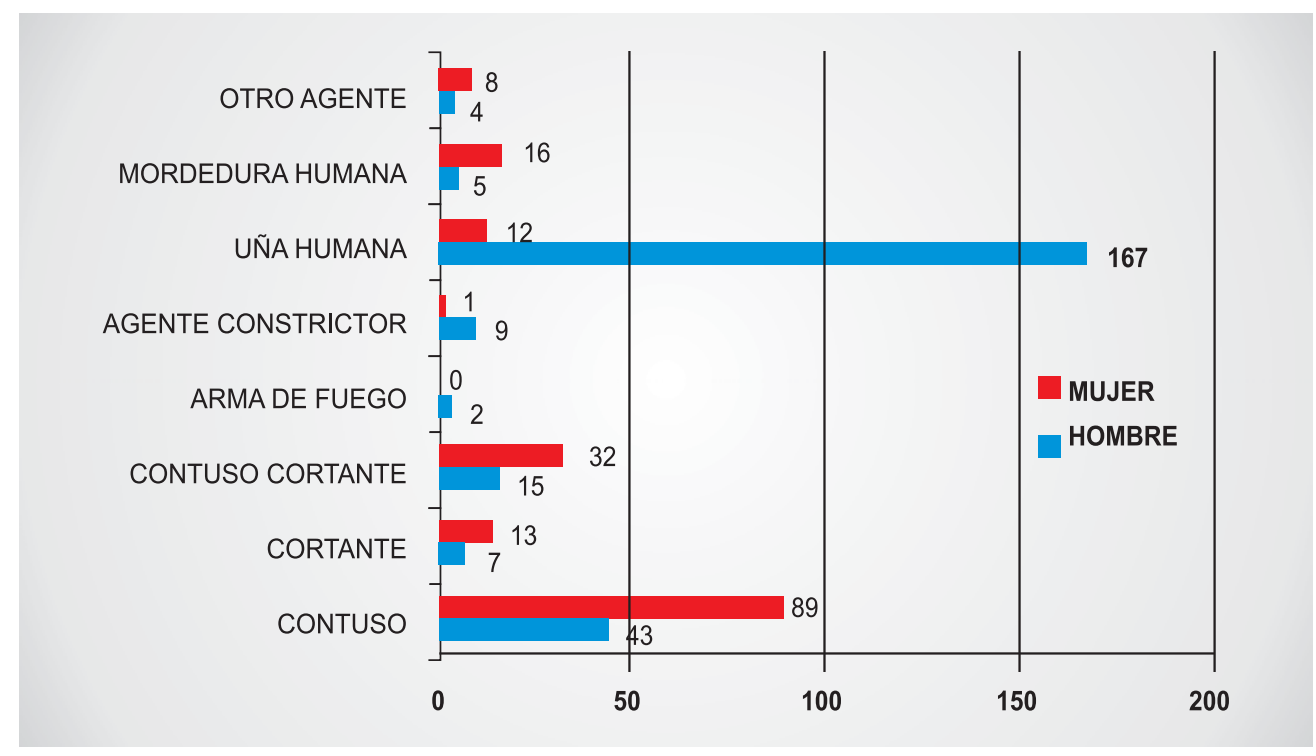

Figura 3. Clasificacion de las lesiones según el agente que las produce

Se determinó según el estado civil y el sexo de los peritados, que los casados y los convivientes son quienes tienen mayor frecuencia de lesiones por violencia familiar, lo expresa que en mujeres el 45,63\% (193/423) de las peritadas era casada, seguido del $16,55 \%(70 / 423)$ eran convivientes. En los peritados de sexo masculino que vinieron por lesiones por violencia familiar el 23,40\% (99/423) son casados y el 3,55\% (15/423) eran convivientes. (Tabla 3, Figura 4).
Se evidenció que las regiones anatómicas lesionadas con mayor frecuencia tanto en peritados varones y mujeres son cabeza y cuello, brazos y tronco. Siendo asi en varones se expresó el $8,75 \%$ (37/423) tuvo lesiones en cabeza y cuello, seguido de $8,27 \%(35 / 423)$ presento lesiones en brazo derecho y el 8,04\% (34/423) presentó lesiones en brazo izquierdo. Pero en el sexo femenino el 9,69\% (41/423) presentó lesiones en cabeza y cuello, seguido de $8,98 \%$ (38/423) presentó lesiones en cara posterior de tronco y el 8,75\% (37/423) presentó lesiones en brazo izquierdo. (Tabla 4). Figuras 5-11.

Tabla 3. Distirbucion de victimas de violencia Familiar según estado civil y sexo.

\begin{tabular}{|c|c|c|c|c|c|}
\hline LESIONES PO & $\begin{array}{l}\text { NCIA FAMI } \\
\text { Masculino }\end{array}$ & $\begin{array}{l}\text { GÚN D } \\
\%\end{array}$ & $\begin{array}{l}\text { BUUCIÓN P } \\
\text { Femenino }\end{array}$ & $\begin{array}{l}\text { KO Y E } \\
\%\end{array}$ & $\begin{array}{r}\text { CIVIL } \\
\text { Total }\end{array}$ \\
\hline Casado & 193 & 45.63 & 99 & 23.40 & 292 \\
\hline Casado separado & 2 & 0.47 & 0 & 0.00 & 2 \\
\hline Conviviente & 70 & 16.55 & 15 & 3.55 & 85 \\
\hline Divorciado & 2 & 0.47 & 0 & 0.00 & 2 \\
\hline Ex-conviviente & 2 & 0.47 & 0 & 0.00 & 2 \\
\hline Soltero & 12 & 2.84 & 14 & 3.31 & 26 \\
\hline Viudo & 12 & 2.84 & 2 & 0.47 & 14 \\
\hline Total & 293 & 69.27 & 130 & 30.73 & 423 \\
\hline
\end{tabular}




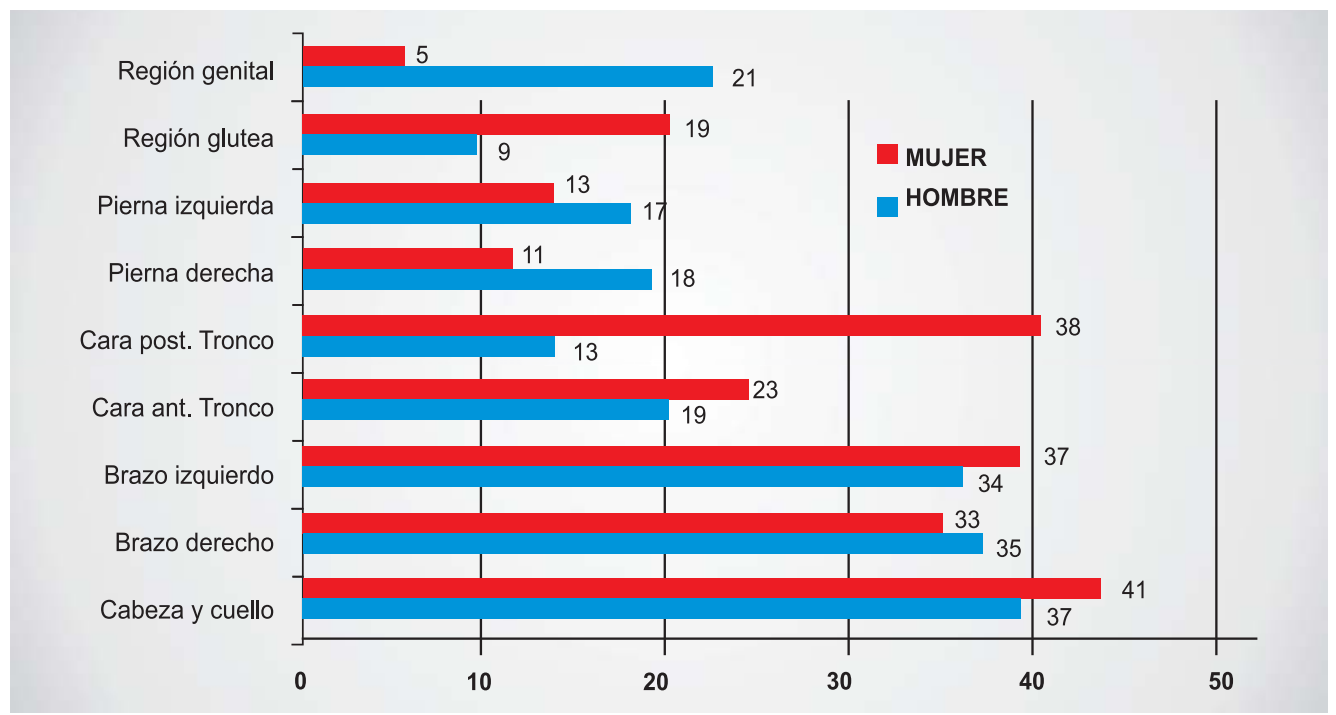

Figura 4.Clasificacion de las lesiones en Violencia Familiar, según la zona del cuerpo afectada y el sexo de la victima.

Tabla 4. Clasificacion de las lesiones en Violencia Familiar, según la zona del cuerpo afectada y el sexo de la victima.

\begin{tabular}{|c|c|c|c|c|}
\hline \multicolumn{5}{|c|}{ ZONA ANATOMICA LESIONADA } \\
\hline & \multicolumn{2}{|c|}{ HOMBRE } & \multicolumn{2}{|c|}{ MUJER } \\
\hline & Número & $\%$ & Número & $\%$ \\
\hline Cabeza y cuello & 37 & 8.75 & 41 & 9.69 \\
\hline Brazo derecho & 35 & 8.27 & 33 & 7.80 \\
\hline Brazo izquierdo & 34 & 8.04 & 37 & 8.75 \\
\hline Cara ant. tronco & 19 & 4.49 & 23 & 5.44 \\
\hline Cara. post. tronco & 13 & 3.07 & 38 & 8.98 \\
\hline Pierna derecha & 18 & 4.26 & 11 & 2.60 \\
\hline Pierna izquierda & 17 & 4.02 & 13 & 3.07 \\
\hline Región glutea & 9 & 2.13 & 19 & 4.49 \\
\hline Region genital & 21 & 4.96 & 5 & 1.18 \\
\hline Total & 203 & 47.99 & 220 & 52.01 \\
\hline
\end{tabular}

\section{DISCUSIÓN}

El RML por violencia familiar o examen médico legal por violencia familiar, es un procedimiento que constituye el acto médico y es una prueba para resolver un problema jurídico.

El RML debe ser redactado técnica y científicamente, de acuerdo al examen solicitado por la autoridad competente; para ello se emplean métodos científicos y técnicos que fuesen posibles, debiendo el profesional que evalúa, obtener la evidencia que se relacione con el delito (equimosis, escoriaciones, contusiones, etc.).

El examen médico legal siempre será practicado, previo consentimiento de la víctima, exclusivamente por el médico encargado con presencia de un personal auxiliar.

Los resultados encontrados en el presente estudio de lesiones por violencia familiar es en sí misma 
un hallazgo importante que coincide con estudios previos sobre el problema $(12,13)$ y constituye un reto que se debe superar por el bien de la funcionabilidad de la familia.

Las mujeres presentan lesiones por agente contuso debido a la fuerza que la caracterizada los agresores que muchas veces son varones (convivientes y esposos) y en el caso de los varones son lesiones por uña humana que caracteriza a las mujeres que muchas veces tras la agresión, usan mecanismos de defensa y crean lesiones por uña humana, además de lesiones contusas concomitantes.

Hasta el momento la violencia familiar es un tema que no ha sido abordado directamente en los aspectos que se requiere trabajar de manera inmediata, es entonces, precisamente, la escasa y equívoca información respecto al tema.

En ese sentido, sería enriquecedor profundizar en el aspecto del desconocimiento generalizado, pues no se satisfacen aún las necesidades de identificación, manejo y control de daños a la salud de las víctimas de violencia familiar.

Lo que se intenta es que reconozcan la magnitud, cualidades y repercusiones del problema, y la oportunidad que tienen para apoyar a las víctimas de violencia familiar en la búsqueda de soluciones a su problema, ya que, tarde o temprano, éstas deterioran el entorno familiar y crearán problemas y disfuncionalidad de la familia.

Resulta necesario considerar que el proceder del personal Médico Legal tendrá impacto sobre el desempeño legal y la mejora del entorno familiar como de la calidad de vida de las víctimas de violencia familiar que son maltratadas por su pareja.

Así entonces podemos concluir, que uno de los mitos que hay que superar, es que la Violencia familiar sólo forma parte de los conflictos de pareja.

Está demostrado que el maltrato dentro de la familia se extiende a sus hijos, afectando negativamente su bienestar y su desarrollo, con secuelas a largo plazo; Ilegando incluso a transmitirse a sucesivas generaciones.

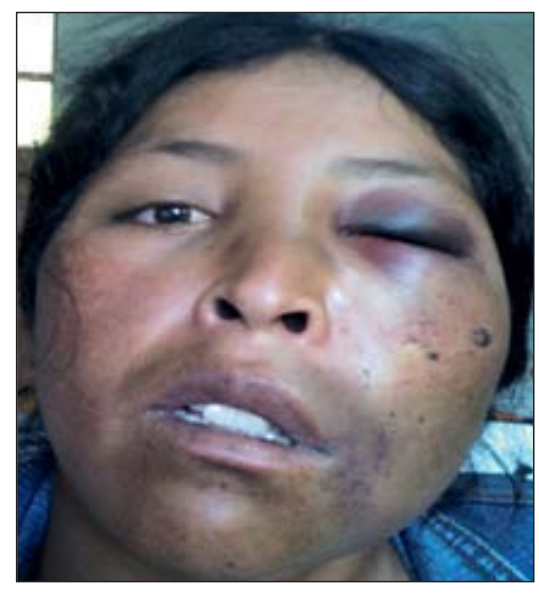

Figura 5. Se observa Hematoma localizado en región palpebral y región infra orbicular izquierda. Tumefacción en tercio medio de pirámide nasal. Esta lesión fue ocasionada por un agente contuso (puño del agresor).

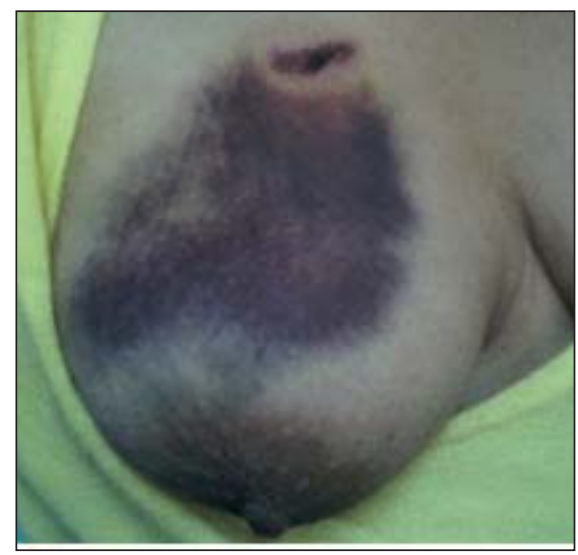

Figura 6. Se observa Equimosis Violácea localizada en mama derecha, dicha equimosis abarca cuadrante superior e inferior derecho e izquierdo de mama derecha; y en el cuadrante superior interno de mama derecha se observa lesión producida por mordedura humana.

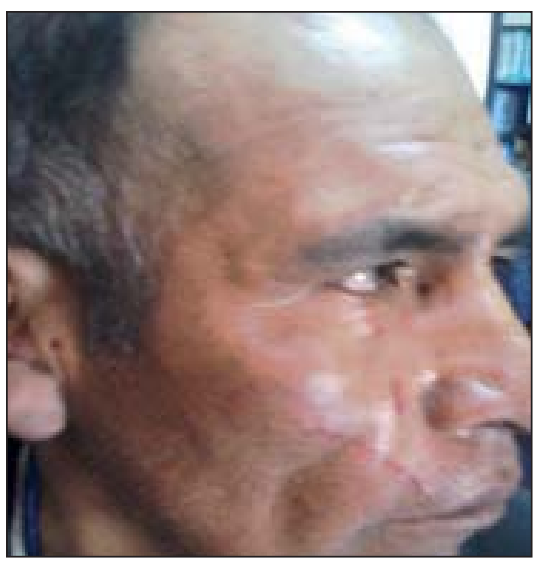

Figura 7. Se observa escoriación lineal localizado desde región infra orbicular derecha hasta mejilla derecha, y otra escoriación lineal que cruza desde región maxilar superior derecha hasta comisura labial derecha. Lesión producida por uña humana. 


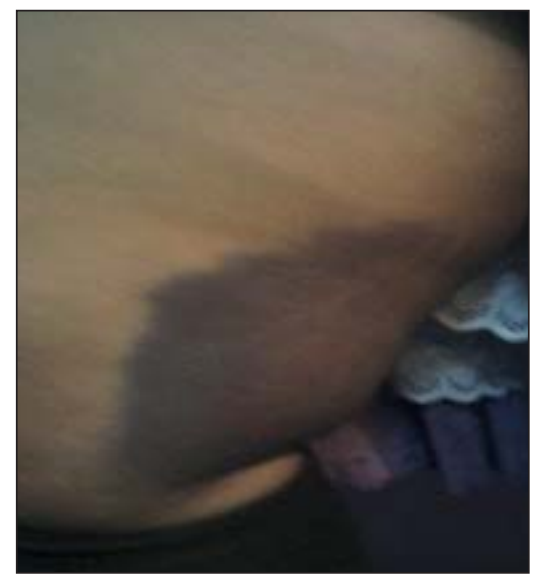

Figura 8. Se observa Equimosis Violácea localizada en cara interna de tercio medio de pierna derecha, típicas de agentes contusos compatibles con patadas (forma casi exacta dela suela del zapato).

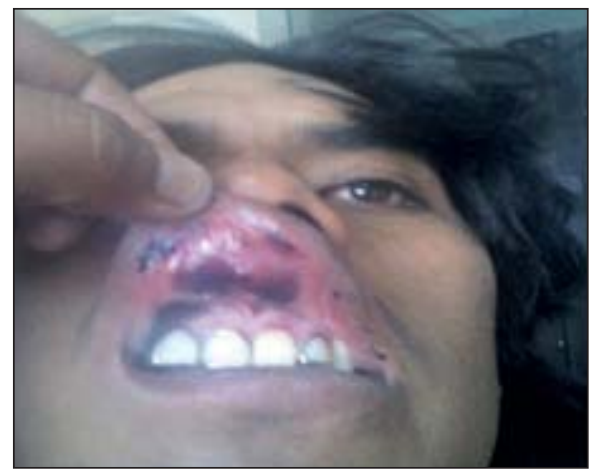

Figura 10. Escoriación y herida abierta localizado en región cigomática izquierda .lesión producida por agente uña humana y fricción.

\section{REFERENCIAS BIBLIOGRÁFICAS}

1. Bonnet EPF, Medicina Legal y Toxicología. Buenos Aires: Libreros, 1980.

2. Calabuig G, Villanueva E. Lesiones. Medicina Legal y Toxicología. $5^{\text {a }}$ Ed. Masson, Barcelona, 1998.

3. Código de Procedimientos Penales. Ley 4019. Art. 161. Nombramiento de Peritos. Preferencia y honorarios. Lima, Perú (23 de noviembre del 1939).

4. Código Penal. Decreto Legislativo N. ${ }^{\circ}$ 635. Art. 121-122. Delitos contra la vida, el cuerpo y la salud. Lesiones.; Perú, 1991.

5. Chalk R, King P. Assesing family violence interventions. Am J Prev Med 1998; 14(4):289-292.

6. Diario Oficial de la Federación. Norma Oficial Mexicana NOM190-SSA1-1999. Prestación de servicios de salud. Criterios para la atención médica de la violencia familiar. México, DF: Secretaría de Salud; 2000.

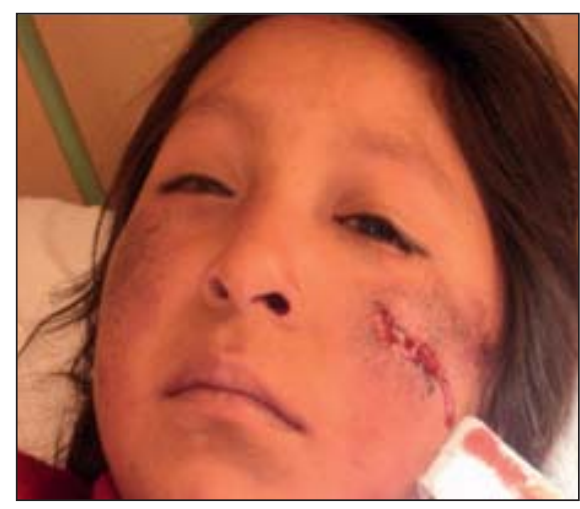

Figura 9. Hematoma en periodo de reabsorción localizado en cara interna (mucosa) de labio superior .lesión producida por agente contuso duro.

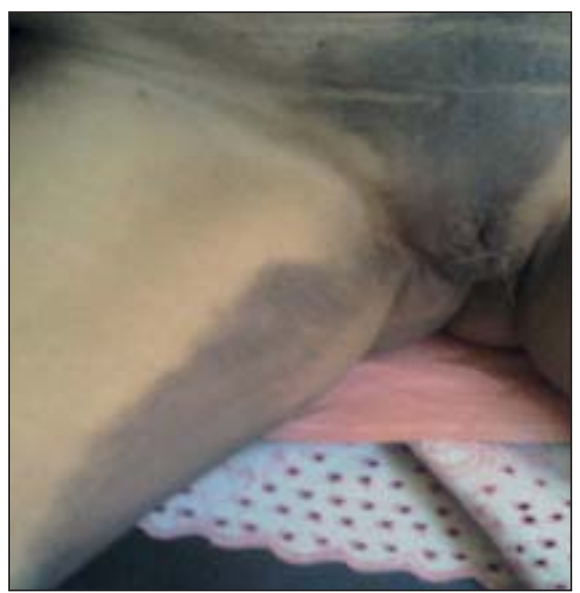

Figura 11.-. Se observa equimosis violáceo localizado en región de hipogastrio y en cara interna de tercio proximal de pierna derecha.
7. Di Maio JM, Dana SE Homicidios relacionados con el sexo. En: Manual de patología forense. Ed. Díaz Santos, Madrid, 2003: 165167.

8. Gayet J, Manual de la Policía Científica. España: Zeus, 1965.

9. Glasser D, Stephen F. Abuso sexual en niños. Ed. Paidós, Buenos Aires, 1997.

10. Gómez de Terreros I: Los profesionales de la salud ante el maltrato. $2^{\mathrm{a} e d .}$ Editorial Comares. Granada, 1997.

11. Guzmán C. Manual de Criminalística, Ediciones LA ROCA, $1^{\text {a }}$ edición, Buenos Aires-Argentina, 2000, p. 487.

12. Gómez de Terreros I: Los profesionales de la salud ante el maltrato. $2^{\mathrm{a}}$ ed. Editorial Comares. Granada, 1997.

13. Harwell TS, CastenRJ, Armstrong KA, Demsey S, Coons HL. Results of a domestic violence training program offered to the staff of 
urban community health centers. Evaluation Committee of the Philadelphia Family Violence Working Group. Am J Prev Med 1998; 153:235-242.

14. Hernández D. Pobreza urbana y violencia doméstica en hogares de la Ciudad de México. ActaSociol. 1998; 22:25-43.

15. Híjar MC, Lozano R, Valdez R, Blanco J. Lesiones intencionales como causa de demanda de atención en servicios de urgencia hospitalaria en la Ciudad de México. Salud Mental 2002; 25(1):3542.

16. Kellermann AL. Domestic violence and the internist's response: Advocacy or apathy? J G Intern Med 1990; 5:89-90.

17. Knight B. Delitos sexuales. En su: Medicina Forense de Simpson. Ed. El Manual Moderno, México, 1994.

18. Kvitko LA. La violación. Peritación médico legal en las presuntas víctimas del delito. 2a edición. México: Trillas 1995.

19. McLeer SV, Anwar R. A study of battered women presenting in an emergency department. Am J Public Health. 1989.

20. Moreno R. "Manual de Introducción a La Criminalística”, Editorial Porrúa S. A., México, 1990.

21. Muñiz M, Ferrer D, González J, Jiménez Y. Violencia intrafamiliar: su presentación en un área de salud. Rev Cubana Med Gen Integr 2000.

22. Parsons LH, Zaccaro D, Wells B, Stoval TG. Methods of and attitudes toward screening obsterics and gynecology patients for domestic violence. Am J ObstetGynecol, 1995.

23. Policía Nacional del Perú: Manual de Criminalística, Servicios Gráficos JMD, Lima-Perú, 2006.

24. Rodríguez H. Abuso sexual en niños: enfoque médico-legal. En Baráibar R (Coord.) La salud en la infancia y la adolescencia. Ed. Arena, Montevideo, 1999.

25. Simonin C. Medicina Legal Judicial. Barcelona: Jims. 1962.

26. Sosa M, Juventino. Criminalística. México D.F.: Limusa, 2002. T. 1. p. 49.

27. Sugg NK, Inui T. Primary care physicians' response to domestic violence. Opening Pandora's Box. JAMA 1992;267(23):3157-3160.

28. UNICEF:Innocenti Report Card Digest $n^{\circ}$ 2. Niños y Violencia. 1999.

29. Valdez R, Juárez C. Impacto de la violencia doméstica en la salud mental de las mujeres: análisis y perspectivas en México. Salud Mental. 1998.

30. Vanrell P. Violencia sexual. En su: Sexología forense. EditoraUnimontes, Montes Claros, 2001.

31. Vargas Alvardo E.Medicina Forense y Deontologia Medica "2da edición. Mexico: Editorial Trillas; 1999.

32. Zajaczkowski R. "Manual de Criminalística", Ediciones Ciudad Argentina, Bs. Aires-Argentina, 1998.

\section{Fuentes de financiamiento}

Este estudio ha sido autofinanciado por el autor.

\section{Conflicto de interés}

\section{El autor declara no tener ningún conflicto de} interés

\section{Correspondencia:}

Ulises Mejía Rodríguez

Dirección: Av. Alfonso Ugarte $N^{\circ} 890$ 1er Piso

Chucuito Juli - Puno. DML I Chucuito Juli.

Teléfono: 942161374

Correo electrónico: ulisesmejiarodriguez@hotmail.com 